\title{
Analysis of the Relationship between Random Telegraph Signal and Negative Bias Temperature Instability
}

\author{
Yasumasa Tsukamoto ${ }^{1,2}$, Seng Oon $\mathrm{Toh}^{2}$, Changhwan $\mathrm{Shin}^{2}$, Andrew Mairena ${ }^{2}$, \\ Tsu-Jae King Liu ${ }^{2}$ and Borivoje Nikolić ${ }^{2}$ \\ ${ }^{1}$ Renesas Technology Corp., Japan. ${ }^{2}$ University of California at Berkeley, USA \\ Email address: tsukamot@eecs.berkeley.edu, tsukamoto.yasumasa@renesas.com
}

\begin{abstract}
Random telegraph signal (RTS) is shown to be an intrinsic component of the shift in MOSFET threshold voltage $\left(V_{\text {th }}\right)$ due to bias temperature instability (BTI). This is done by starting from a well-known model for negative BTI (NBTI), to derive the formula for RTS-induced $V_{\text {th }}$ shift. Based on this analysis, RTS simply contributes an offset in NBTI degradation, with an acceleration factor that is dependent on the gate voltage and temperature. This is verified by 3-dimensional (3-D) device simulations and measurements of $45 \mathrm{~nm}$-node bulk-Si PMOS transistors. It has an important implication for design of robust SRAM arrays in the future: design margin for RTS should not be simply added, because it is already partially accounted for within the design margin for NBTI degradation.
\end{abstract}

Keywords-component; random telegraph signal; negative bias temperature instability; hole traps; SRAM

\section{INTRODUCTION}

Random telegraph signal (RTS) is a temporal variation in $I_{d s}\left(V_{t h}\right)$ caused by the capture and emission of mobile charge carriers, and it has been studied for over two decades [1-6]. Since the amplitude of RTS is proportional to the inverse of the MOSFET channel area [3], memory devices manufactured using the most advanced fabrication technologies are facing severe challenges due to this phenomenon. Being different from flash memory devices which have thick gate-oxide films, SRAM devices until recently were not considered to be seriously affected by RTS because they utilize thin gate-oxide films in accordance with MOS scaling [7]. However, because $V_{t h}$ variation due to RTS eventually (with scaling) will become comparable to that due to random dopant fluctuations (RDF) [5] - a major source of degradation in SRAM characteristics [8] -studies of the impact of RTS on highly scaled SRAM have been reported [9-12]. Although it is still controversial how much the SRAM minimum operation voltage $\left(\mathrm{V}_{\min }\right)$ is degraded by RTS, SRAM designers should allocate some design margin for RTS in addition to a (larger) design margin for RDF. Furthermore, degradation in PMOS $\mathrm{V}_{\text {th }}$, i.e. negative bias temperature instability (NBTI) [13-15], should be considered. With the advent of high-k/metal-gate transistors in SRAM, $V_{\min }$ degradation due to positive bias temperature instability (PBTI) will also be significant $[16,17]$.

There is therefore a need to establish appropriate design margins to guarantee sufficient SRAM yield for operation at $\mathrm{V}_{\text {min }}$, at minimum cost (i.e. without overdesigning the cells). In this regard, it is necessary to account for inter-dependencies between the physical phenomena that cause $\mathrm{V}_{\text {th }}$ variation and degradation. Islam et al. introduced a model for NBTI comprising two components, associated with hole traps and with interface traps $[18,19]$. The effect of hole trapping appears for short stress times and eventually saturates at some constant value. On the other hand, interface trapping (attributed to dangling $\mathrm{Si}-\mathrm{H}$ bonds at the $\mathrm{Si}-\mathrm{SiO}_{2}$ boundary) follows a power-law relationship with stress time and is the dominant component of $V_{t h}$ degradation for long stress times. This behavior has been observed experimentally [20], so it is recognized to be a plausible explanation of the NBTI effect. Meanwhile, RTS has been explained to be due to hole trapping/detrapping, and therefore should be related to NBTI. In fact, Kaczer et al. pointed out a broad similarity between the relaxation process of NBTI and 1/f noise [21]. Furthermore, Grasser et al. succeeded in reproducing the time constants of RTS by using a recovery model of NBTI [22], which indicates that care must be taken to properly account for these two phenomena for worst-case SRAM design margins.

In this paper, a relationship between the $V_{t h}$ degradation of RTS and that of NBTI is established, to allow for minimization of design margin. This paper is organized as follows. In section II, the relationship between RTS- and NBTI-induced $V_{\text {th }}$ degradation is derived from theory. In section III, the theory is verified by analyzing device simulation results and some measured data for SRAM fabricated using 45nm CMOS technology. Finally, in Section IV, conclusions are drawn.

\section{THEORETICAL ANALYSIS}

$V_{\text {th }}$ degradation due to NBTI is modeled as the sum of an interface-trap effect $\left(\Delta V_{\mathrm{IT}}\right)$ and a hole-trap effect $\left(\Delta V_{\mathrm{H}}\right)[18]$ :

$$
\begin{aligned}
\Delta V_{t h} & =\Delta V_{I T}+\Delta V_{H} \\
& =a \frac{q \Delta N_{I T}(t)}{C_{o x}}+\frac{\int_{0}^{T o x} \int_{E} x \rho_{H}(x, E, t) d E d x}{C_{o x} T_{o x}}
\end{aligned}
$$

where $a$ is the fraction of donor-like traps, $\Delta N_{\text {IT }}$ is the areal density of interface traps, $C_{\mathrm{ox}}$ is the areal gate capacitance, $T_{\mathrm{ox}}$ is the gate oxide thickness, and $\rho_{\mathrm{H}}$ is the areal charge density due to trapped holes in the gate oxide, respectively. The first term is dominant at long stress times, and shows a power-law relationship with stress time $t$; it is proportional to $t^{n}$, where $n$ ranges from $1 / 6$ to $1 / 3$. The second term saturates at some 
value within a relatively short time (milliseconds). According to [18],

$$
\rho_{H}\left(x, E_{i}, t\right)=N_{0} \delta\left(E-E_{i}\right) f_{T}\left(x, E_{i}, t\right)
$$

where $N_{0}$ is the areal density of pre-existing traps within the gate oxide and $E_{\mathrm{i}}$ is the energy level of the trap. $f_{\mathrm{T}}$ is the probability that a hole trap is filled, which evolves with time:

$$
\frac{d f_{T}}{d t}=\sigma \cdot v_{t h} \cdot\left[p_{h} T_{1}\left(1-f_{T}\right)-n_{S} T_{1} f_{T}-n_{G} T_{2} f_{T}\right]
$$

where $\sigma$ is the capture cross-section, $v_{\text {th }}$ is the thermal velocity, $p_{\mathrm{h}}$ is the inversion-layer hole areal density, and $n_{\mathrm{S}}$ and $n_{\mathrm{G}}$ are the areal concentrations of states in the substrate and in the gate, respectively. $T_{1}$ and $T_{2}$ are the probabilities for tunneling between the substrate and the gate oxide, and for tunneling between the gate oxide and the gate, respectively. This equation was solved in [18] for the initial condition $f_{\mathrm{T}}(0)=0$, i.e. zero initially trapped holes. Taking RTS into consideration, however, $f_{\mathrm{T}}(0)$ should have a non-zero value. We therefore propose to separate $f_{T}$ into a shallow-trap term $f_{\text {T- }}$ $\mathrm{s}$ and a deep-trap term $f_{\mathrm{T}-\mathrm{D}}$, and subsequently focus on $f_{T-S}$ since the derivation of $f_{\mathrm{T}-\mathrm{D}}$ is the same as that given in [18]. From [23], $f_{\mathrm{T}}=\tau_{\mathrm{e}} /\left(\tau_{\mathrm{e}}+\tau_{\mathrm{c}}\right)$ under equilibrium conditions, where $\tau_{\mathrm{c}}$ is the mean time to hole capture and $\tau_{\mathrm{e}}$ is the mean time to hole emission. This is the appropriate initial value for $f_{\mathrm{T}-\mathrm{S}}$. Note that $\tau_{\mathrm{e}} /\left(\tau_{\mathrm{e}}+\tau_{\mathrm{c}}\right)$ is the average duty cycle for hole trapping: if the hole trap is mostly filled $\left(\tau_{\mathrm{e}} \rightarrow \infty\right)$, it approaches 1 ; if the hole trap is mostly empty $\left(\tau_{c} \rightarrow \infty\right)$, it approaches 0 . The solution to (3) for the shallow-trap term is therefore

$$
\begin{aligned}
f_{T-S}(t)= & \frac{T_{1}}{\left(1+\frac{n_{S}}{p_{h}}\right) T_{1}+\frac{n_{G}}{p_{h}} T_{2}}\left[1-\exp \left(-\frac{t}{\tau_{N B T I}}\right)\right] \\
& +\frac{\tau_{e}}{\tau_{e}+\tau_{c}} \exp \left(-\frac{t}{\tau_{N B T I}}\right)
\end{aligned}
$$

where $1 / \tau_{\mathrm{NBTI}}=\sigma v_{\mathrm{th}}\left[\left(p_{\mathrm{h}}+n_{\mathrm{S}}\right) T_{1}+n_{\mathrm{G}} T_{2}\right]$. It should be noted that the last term in (4) does not appear in the expression for $f_{\mathrm{T}-\mathrm{D}}$ [18]. Based on the Wentzel-Kramers-Brillouin (WKB) approximation, it can be assumed that $T_{1} \gg T_{2}$ if the trap is located closer to the channel than to the gate. Since $n_{\mathrm{s}}=1 / \sigma v_{\mathrm{th}} \tau_{\mathrm{e}}$ and $p_{\mathrm{h}}=1 / \sigma v_{\mathrm{th}} \tau_{\mathrm{c}}$ [23], the saturated value of $f_{\mathrm{T}-\mathrm{S}}$ is simply given by

$$
f_{T-S}(t)=\frac{\tau_{e}}{\tau_{e}+\tau_{c}} .
$$

Assuming that the hole traps are located at a distance $z$ from the substrate-oxide interface, $N_{0}=\left(q /\left(L_{\text {eff }} W_{\text {eff }}\right)\right) \cdot \delta\left(x-\left(T_{\text {ox }}-z\right)\right)$. The integral for $\Delta V_{\mathrm{H}}$ can then be evaluated:

$$
\left.\Delta V_{H}\right|_{t \rightarrow \infty}=\frac{q}{L_{e f f} W_{e f f} C_{o x}}\left(1-\frac{z}{T_{o x}}\right) \frac{\tau_{e}}{\tau_{e}+\tau_{c}} \equiv \Delta V_{R T S} \frac{\tau_{e}}{\tau_{e}+\tau_{c}} .
$$

Note that $\Delta V_{\mathrm{RTS}}=\left(q /\left(L_{\mathrm{eff}} W_{\mathrm{eff}} C_{\mathrm{ox}}\right)\right) \cdot\left(1-z / T_{\mathrm{ox}}\right)$ is the expression for the $V_{\text {th }}$ shift due to RTS derived in [3] in a different manner, and that $\tau_{\mathrm{e}} /\left(\tau_{\mathrm{e}}+\tau_{\mathrm{c}}\right)$ is the average duty cycle for hole trapping. Therefore, the expression in (6) represents the timeaveraged value of $V_{\text {th }}$ shift due to RTS. Substituting (6) into (1), the total $V_{\text {th }}$ degradation due to NBTI is expressed by

$$
\Delta V_{t h}(t)=\Delta V_{R T S} \frac{\tau_{e}}{\tau_{e}+\tau_{c}}+A\left(1-\exp \left(-\frac{t}{\tau_{N B T I}}\right)\right)+B t^{n}
$$

where $A$ and $B$ are constants. In (7), the first term corresponds to shallow traps, the second to deep traps, and the third to interface traps. Considering that actual NBTI measurements of $V_{\text {th }} v s . t$ can be fitted to the empirical model $V_{\text {th }}=C+D t^{n}$ [20], the first term is included in $C$, i.e. the effect of RTS is included in NBTI degradation.

The relationship between RTS and NBTI is clarified further by examining the factor $\tau_{e} /\left(\tau_{\mathrm{c}}+\tau_{\mathrm{e}}\right)=1 /\left(1+\tau_{\mathrm{c}} / \tau_{\mathrm{e}}\right) . \quad \tau_{\mathrm{c}} / \tau_{\mathrm{e}}$ is dependent on the gate voltage $V_{\mathrm{g}}$ and absolute temperature $T$, decreasing with $V_{\mathrm{g}}$ and increasing with $T$ [3]:

$$
\frac{\tau_{c}}{\tau_{e}}=\exp \left(K-\frac{q}{k T}\left[\left(1-\frac{z}{T_{o x}}\right) \Psi_{S}+\frac{z}{T_{o x}} V_{g}\right]\right) .
$$

where $K$ is a constant and $\Psi_{\mathrm{S}}$ is the channel surface potential. Generally, RTS is measured under normal operating conditions $\left(V_{\mathrm{g} 0}, T_{0}\right)$ whereas NBTI is measured under accelerated stress conditions $\left(V_{\mathrm{g} 0}+\Delta V, T_{0}+\Delta T\right)$, so that one must be careful to account for $\Delta V$ and $\Delta T$ when correlating the first term in (7) with RTS. This can be done by introducing the concept of an acceleration factor $\alpha(\Delta V, \Delta T)$ :

$$
\frac{\Delta V_{R T S}}{1+\left.\left(\tau_{c} / \tau_{e}\right)\right|_{\left(V_{g 0}+\Delta V, T_{0}+\Delta T\right)}} \equiv \Delta V_{R T S} \cdot \alpha(\Delta V, \Delta T) .
$$

Note that (7) deals with a single-trap RTS with depth $z$ where both $\Delta V_{\text {RTS }}$ and $\alpha$ are the function of $z$. Therefore we can expand (7) to include the multiple traps with depths $z_{i}$ (where $i$ represents the trap number) as,

$$
\Delta V_{t h}(t)=\sum_{i} \Delta V_{R T S}^{i} \cdot \alpha_{i}+A\left(1-\exp \left(-\frac{t}{\tau_{N B T I}}\right)\right)+B t^{n}
$$

The dependence of $\tau_{\mathrm{c}} \tau_{\mathrm{e}}$ on $V_{\mathrm{g}}$ is illustrated in the representative example shown in Fig. 1(a), for which the normal operating conditions are $(1.0 \mathrm{~V}, 300 \mathrm{~K})$ and the NBTI stress conditions are $(2.0 \mathrm{~V}, 400 \mathrm{~K})$. It can be seen that $\tau_{\mathrm{c}} / \tau_{\mathrm{e}}=1$ under normal operating conditions, so that $\alpha=50 \%$ for $(\Delta V$, $\Delta T)=(0 \mathrm{~V}, 0 \mathrm{~K})$ in Fig. 1 (b). In contrast, $\tau_{\mathrm{c}} / \tau_{\mathrm{e}} \approx 0.01$ under NBTI stress conditions, so that $\alpha \approx 100 \%$ for $(\Delta V, \Delta T)=(1.0 \mathrm{~V}$, $100 \mathrm{~K})$. Thus, under NBTI stress conditions, $100 \%$ of $\Delta V_{R T S}$ appears as an offset in the NBTI-induced $V_{\text {th }}$ shift. However, when the stress is removed, $\alpha$ is decreased to its normal value. This implies that the traps responsible for RTS are also responsible for the NBTI recovery as reported in [21, 22]. Note that Fig. 1 is a sample using $z / T_{o x}=0.25$ and $K=18.35$ condition and thus, $\alpha$ also depends on the values of $z / T_{o x}$ and $K$; small $z$ and large $K$ result in large $\tau_{\mathrm{c}} / \tau_{\mathrm{e}}$ (since $V_{\mathrm{g}}>\Psi_{\mathrm{S}}$ ) and hence small $\alpha$. 

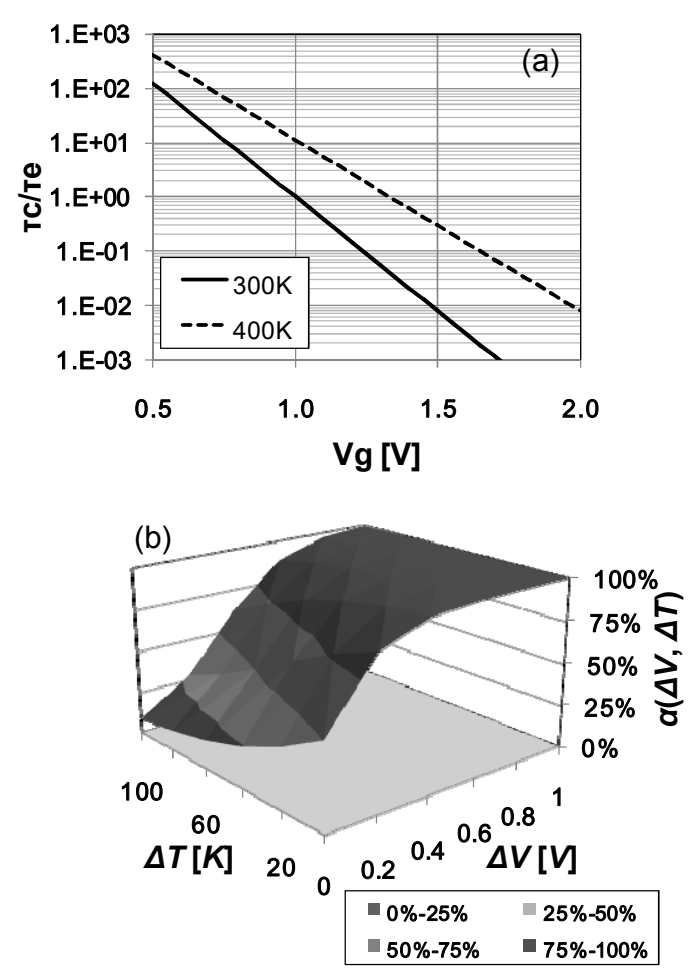

Figure 1: $V_{g}$ and $T$ dependence of $\tau_{c} / \tau_{e}$ (a) and of the acceleration factor (b). The origin $(0,0)$ in (b) corresponds to the nominal operating condition while $(1,100)$ corresponds to the NBTI stress condition.

\section{VALIDATION OT THE THEORY}

Both 3-D device simulations using Sentaurus v.2009.06 (Synopsys, Inc.) and measurements of RTS and NBTI-induced $V_{\text {th }}$ shifts for pull-up devices in 45nm-node SRAM cells [12] were performed in order to validate the theory as developed above. Fig. 2 shows the 3-D device simulation results for a pchannel MOSFET $\left(L / W=60 \mathrm{~nm} / 60 \mathrm{~nm}, T_{\text {ox }}=2 \mathrm{~nm}\right)$ which has a single trap located at the center of the channel area and a distance $0.1 \mathrm{~nm}$ away from the substrate-oxide interface $(z=$ $0.1 \mathrm{~nm})$. The expected dependence of RTS on drain current $\left(I_{\mathrm{D}}\right)[4]$ is reproduced, as shown in Fig. 2. Also, the simulated $\Delta V_{\mathrm{RTS}}$ shows no $T$ dependence (inset), as expected.

Fig. 3 shows the measured dependence of $\tau_{\mathrm{c}} / \tau_{\mathrm{e}}$ on $V_{\mathrm{g}}$ and $T$ for a pull-up transistor in a $45 \mathrm{~nm}$-node bulk-Si SRAM cell. Each $\tau_{\mathrm{c}} / \tau_{\mathrm{e}}$ data point is extracted from the RTS time-domain measurement. Inset table indicates $z / T_{\text {ox }}$ and $K$ values extracted from the slope and $y$-intercept for $304 \mathrm{~K}$ and $337 \mathrm{~K}$ plots, respectively. Those values are almost equivalent to each other, confirming the validity of using (8) at different $V_{g}$ and $T$ conditions. For instance, since $\tau_{\mathrm{c}} / \tau_{\mathrm{e}}$ at $V_{\mathrm{g}}=1.1 \mathrm{~V}$ at $304 \mathrm{~K}$ is 3.997 , we observe $20 \%$ of RTS-induced $V_{\text {th }}$ shift as the meantime value at nominal condition. On the other hand, $\tau_{\mathrm{c}} / \tau_{\mathrm{e}}$ at $V_{\mathrm{g}}$ $=2.0 \mathrm{~V}$ and $T=400 \mathrm{~K}$ is evaluated as $1.124 \mathrm{E}-2$, which projects 0.989 of $\alpha$, i.e. $98.9 \%$ of the RTS-induced $V_{\text {th }}$ shift is expected to be included in the NBTI degradation.

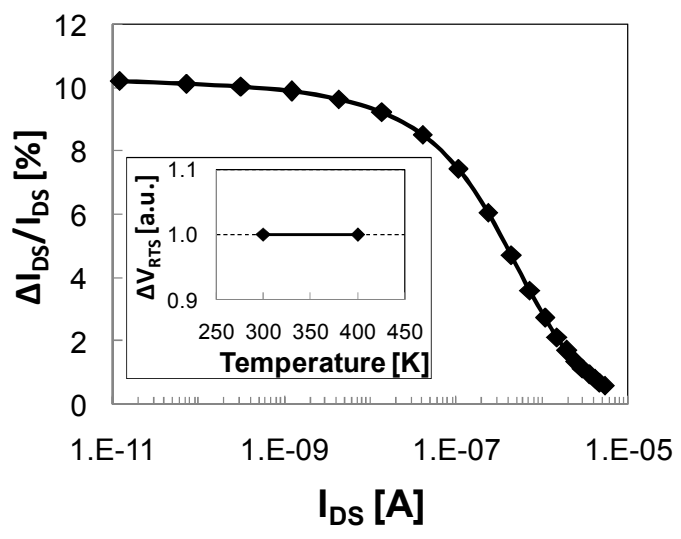

Figure 2: $\Delta I_{\mathrm{DS}} / I_{\mathrm{DS}}$ dependence on $\mathrm{I}_{\mathrm{D}}$ using 3-D device simulation results for a PMOSFET with channel dimensions $L / W=60 \mathrm{~nm} / 60 \mathrm{~nm}$ and uniform body doping $\mathrm{N}_{\mathrm{SUB}}=10^{18} \mathrm{~cm}^{-3}$. Inset shows temperature dependence of $\Delta V_{\mathrm{RTS}}$.

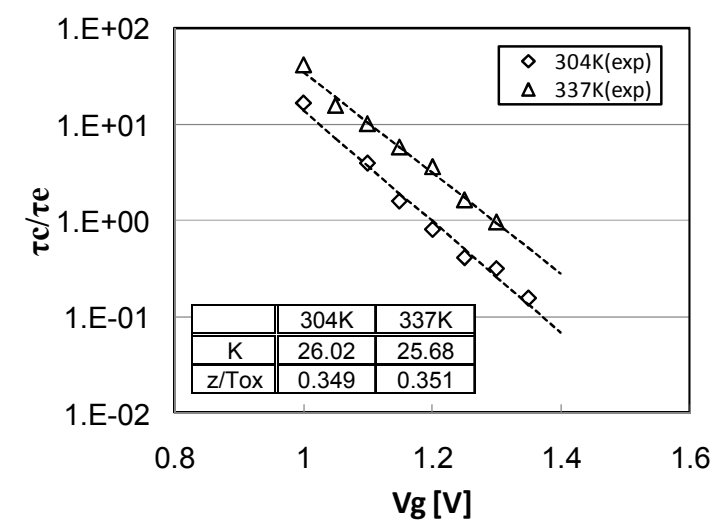

Figure 3: Measured $V_{g}$ and $T$ dependence of $\tau_{\mathrm{c}} / \tau_{\mathrm{e}}$, for a pull-up transistor within a $45 \mathrm{~nm}$-node bulk-Si SRAM cell.

Fig. 4(a) shows measured $V_{\text {th }}$ degradation at $\left|V_{\mathrm{g}}\right|=1.5 \mathrm{~V}$ and $T=400 \mathrm{~K}$ using the on-the-fly (OTF) technique [24, 25]. A prior work reveals that the hole trap effect saturates within 1-s after stress, while the interface trap component starts degrading after 1-s [26]. Based on this fact, $V_{t h}$ degradation during the first second can be entirely attributed to RTS with no interface trap effect. Fig. 4(b) is a close-up graph of Fig 4(a), and it also shows the RTS observed at $V_{\mathrm{g}}=1.1 \mathrm{~V}, T=$ $300 \mathrm{~K}$. Note that (i) the RTS was measured for about $20 \mathrm{sec}$ using the alternating bias method [12], where the time axis is normalized to 1-s scale duration to compare the amplitudes, (ii) the $\Delta V_{t h}=0$ point (bottom line of RTS data) is being shifted upward. From this graph, NBTI behavior can be separated into three phases; first, there is a deep trap effect that appears in the second term of (10) as well as in the fluctuation (Phase 1). Second, there appears the RTS related fluctuation with an amplitude equivalent to that observed at the nominal condition (Phase 2). A rough estimate of the acceleration factor $\alpha$ from the phase 2 is $57 \%$, compared to $23 \%$ for the nominal operating condition, confirming that $\alpha$ is larger for higher $V_{g}$ and $T$. Third, additional RTS fluctuations appear with amplitudes of $\sim 3 \mathrm{mV}$ and $\sim 5 \mathrm{mV}$ in arbitrary units (a.u.), 
equivalent to those observed in Phase 1, by subtracting out the deep trap effect (Phase 3). In summary, for $V_{t h}$ degradation due to NBTI, a deep-trap effect appears during the first 1-s of stress, then RTS (with larger $\alpha$ value than observed for the nominal operating condition) appears, followed by some additional RTS induced by the NBTI stress.
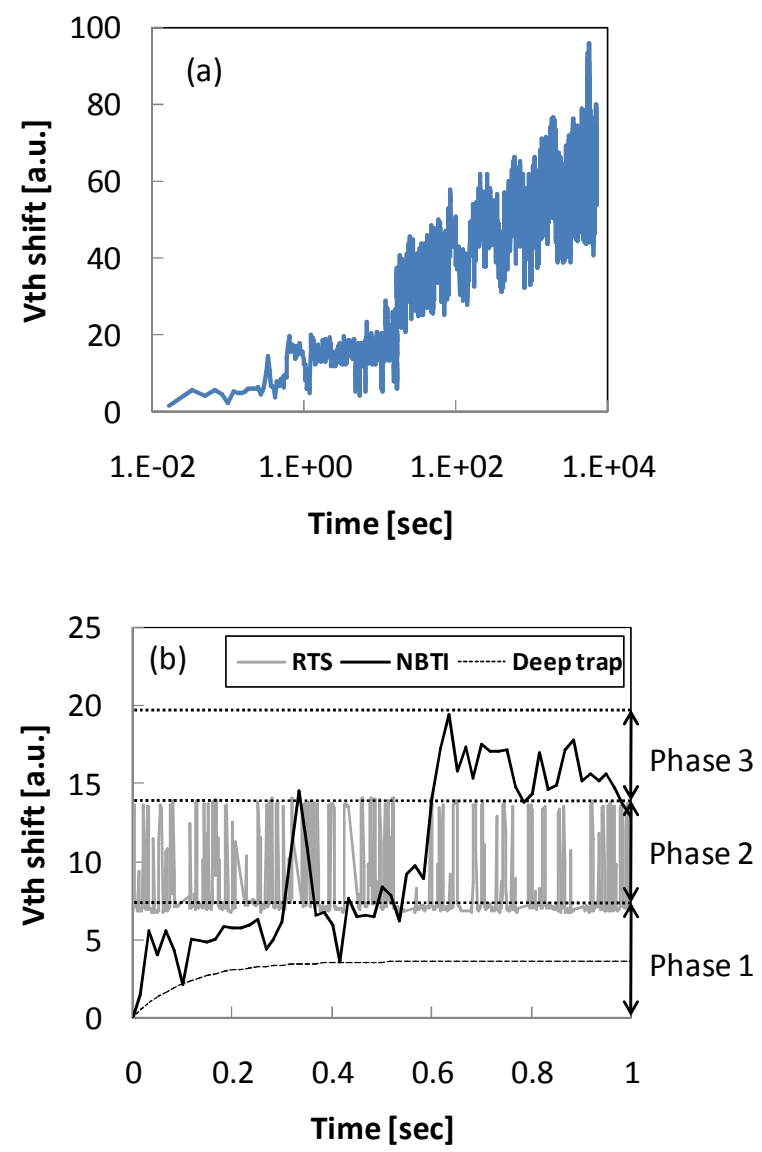

Figure 4: $V_{t h}$ degradation under NBTI stress for long time (a), for 1s after stress (b). In (b), the gray line is the RTS observed for nominal bias condition. Black dashed line indicates deep trap effect which is obtained by fitting $A\left(1-\exp \left(-t / \tau_{N B T I}\right)\right)$ to the local minimum points.

Figure 5(a) shows the $V_{t h}$ shift induced by NBTI for long stress times (from $5000 \mathrm{sec}$ to $7200 \mathrm{sec}$ in Fig. 4(a)) using a time lag plot (TLP) developed by T. Nagumo et al. [27], which was originally used for analyzing RTS. In this method, based on a sequence of $V_{t h}$ shifts represented by $x_{i}(i=$ data number), we generate a two dimensional variable $\left(x_{i}, x_{i+1}\right)$ and plot it on the $x-y$ plane. We use the TLP because it allows us to analyze the RTS amplitude more effectively than using a complex time-domain waveform. Since the NBTI-induced $V_{t h}$ shift includes a time-dependent component associated with the interface trap, the data distributes toward the upper right-hand side compared with the case of RTS analysis [27]. From this figure, three diagonal trend lines can be identified. The difference between those lines is found to be $7 \mathrm{mV}$ (a.u.) and corresponds to the RTS amplitude observed in the NBTI degradation. To see this more effectively, we extract the absolute values of all points measured from the diagonal line in Fig. 5(a) (see Fig. 5(b)). A peak at $7 \mathrm{mV}$ is observed as well as at $5 \mathrm{mV}$, which is related to the RTS observed in Phases 1 and 3 as discussed with regard to Fig 4(b). Regarding the RTS with $3 \mathrm{mV}$ amplitude, it is hard to distinguish a peak in this graph; however, it is noticeable that some large $V_{t h}$ shifts with $\sim 15 \mathrm{mV}$ are observed, which can be explained by coincident RTS with amplitudes of 7,5 and $3 \mathrm{mV}$. Therefore, RTS observed during the first $1 \mathrm{~s}$ of NBTI degradation is retained for long stress times, showing the validity of equation (10).
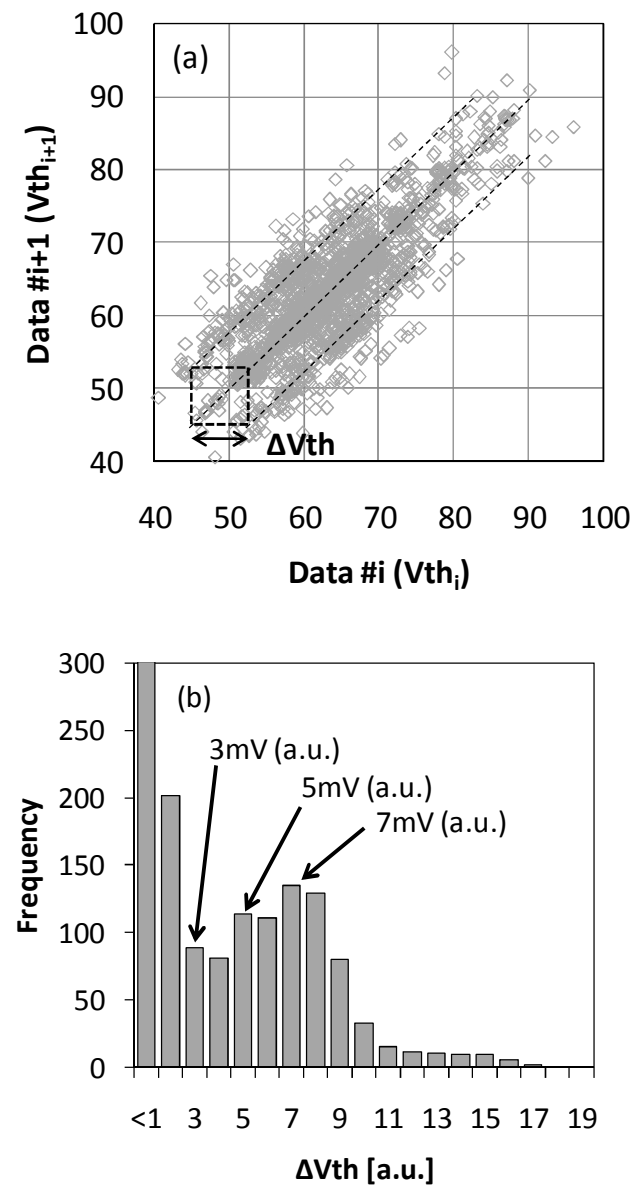

Figure 5: $V_{t h}$ degradation of NBTI during $5000 \mathrm{~s}$ to $7200 \mathrm{~s}$ stress time using TLP metric (a) and the distribution of amplitudes extracted from the diagonal line (b).

To compare the RTS and NBTI data more quantitatively, it is effective to take the time-averaged $V_{t h}$ shift after $1 \mathrm{sec}$ stress because the first and the second terms in (10) represent the time-averaged amplitude. Regarding Fig. 4(b), we obtain $10.15 \mathrm{mV}$ (a.u.) for NBTI and $2.07 \mathrm{mV}$ (a.u.) for RTS, indicating that the time-averaged $V_{\text {th }}$ shift of NBTI has a larger value than that of RTS. We performed the same experiment for multiple transistors in our chip and compared the timeaveraged $V_{t h}$ shift of RTS with that of NBTI (see Fig. 6). All points appear above the diagonal line, confirming that the RTS-induced shift is included in the NBTI-induced shift, validating the theory developed in this work. 


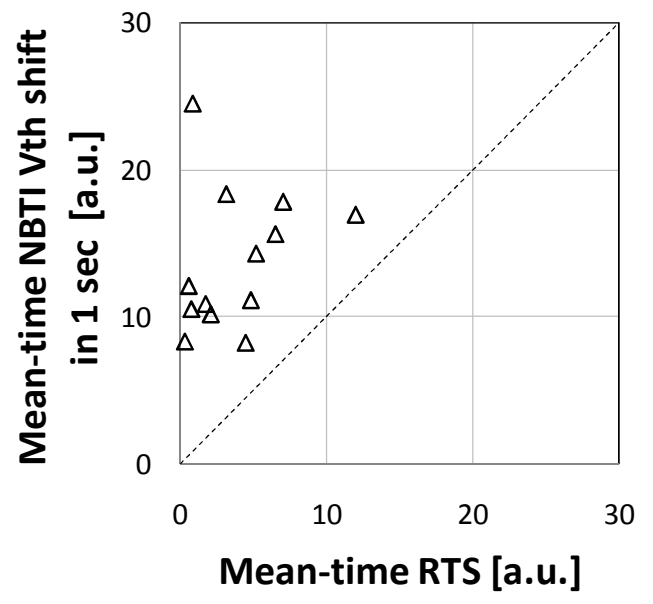

Figure 6: Comparison between mean-time $V_{\text {th }}$ shift of RTS and that of NBTI during $1 \mathrm{sec}$ after applying stress.

\section{CONCLUSION}

The expression for the $V_{\text {th }}$ shift due to RTS is derived starting from a well-known model for $V_{\text {th }}$ degradation due to NBTI. This indicates that the hole traps primarily responsible for the time-independent $V_{\text {th }}$ shift (modeled by the mean-time $V_{\text {th }}$ shift during $1 \mathrm{sec}$ after stress) under NBTI stress are the same ones responsible for RTS. The mean-time $V_{t h}$ shift of NBTI during $1 \mathrm{sec}$ that is manifested as RTS, referred to herein as an acceleration factor $\alpha$, is dependent on the NBTI stress conditions (gate voltage and temperature). 3-D device simulations and SRAM pull-up transistor measurements are consistent with this theory. It can be concluded, therefore, that adding design margin for RTS independently of the design margin for NBTI would result in overly conservative VLSI design.

\section{ACKNOWLEDGMENT}

The authors would like to thank Koji Nii, Makoto Yabuuchi in Renesas Technology Corp., and Zheng Guo in University of California, Berkeley for their technical support and valuable discussions. The first author also thank to Hiroshi Makino in Osaka Institute of Technology and Hirofumi Shinohara, Hisashi Matsumoto, Yuji Kihara, Yohinobu Nakagome and Toshifumi Takeda in Renesas Technology Corp. for their support.

\section{REFERENCES}

[1] G. Ghibaudo, "On the Theory of Carrier Number Fluctuations in MOS Devices," Solid-State Electronics Vol.32, No.7, pp. 563-565, 1989.

[2] K. K. Hung et. al., "Random Telegraph Noise of Deep-Submicrometer MOSFET's," Electron Device Letters, Vol. 11, No. 2, pp. 90-92, 1990.

[3] O. Buisson et. al., "Model for Drain Current RTS Amplitude in SmallArea MOS Transistors," Solid-State Electronics Vol.35, No.9, pp. 1273$1276,1992$.

[4] A. Asenov, et. al., "RTS Amplitudes in Decananometer MOSFETs: 3-D Simulation Study," Transactions on Electron Devices, Vol. 50, No. 3, pp. 839-845, 2003.
[5] K. Abe et. al., "Random Telegraph Signal Statistical Analysis using a Very Large-Scale Array TEG with 1M MOSFETs," Symposium on VLSI Tech. Dig., pp. 210-211, 2007.

[6] J.P. Campbell et. al., "Random Telegraph Noise in Highly Scaled nMOSFETs," IRPS Tech. Dig., pp. 382-388, 2009.

[7] K. Sonoda et. al., "Discrete Dopant Effects on Statistical Variation of Random Telegraph Signal Magnitude," Transactions on Electron Devices, Vol. 54, No. 8, pp. 1918-1925, 2007.

[8] Y. Tsukamoto et. al., "Worst Case Analysis to Obtain Stable Read/Write DC Margin of High Density 6T-SRAM-Array with Local Vth Variability," ICCAD Tech. Dig., pp.398-405, 2005.

[9] M. Agostinelli et. al., "Erratic Fluctuations of SRAM Cache Vmin at the 90nm Process Technology Node," IEDM Tech. Dig., pp. 655-658, 2005.

[10] N. Tega et. al., "Impact of Threshold Voltage Fluctuation due to Random Telegraph Noise on Scaled-down SRAM," IRPS Tech. Dig., pp. 541-546, 2008

[11] K. Takeuchi et. al., "Single-Charge-Based Modeling of Transistor Characteristics Fluctuations Based on Statistical Measurement of RTN Amplitude," Symposium on VLSI Tech. Dig, pp. 54-55, 2009.

[12] S. O. Toh et. al., "Impact of Random Telegraph Signals on $V_{\min }$ in $45 \mathrm{~nm}$ SRAM," IEDM Tech. Dig., pp.767-770, 2009.

[13] J.C. Lin et. al., "Prediction and Control of NBTI - Induced SRAM V ccmin Drift," IEDM Tech. Dig., 2006.

[14] A.T. Krishnan et. al., "SRAM Cell Static Noise Margin and VMIN Sensitivity to Transistor Degradation," IEDM Tech. Dig., 2006.

[15] V. Huard et. al., "NBTI Degradation: From Transistor to SRAM Arrays," IRPS Tech. Dig., pp. 289-300, 2008.

[16] J.C. Lin et. al., "Time Dependent $\mathrm{V}_{\text {ccmin }}$ Degradation of SRAM Fabricated with High-k Gate Dielectrics," IRPS Tech. Dig., pp. 439-444, 2007.

[17] A. Bansal et. al., "Impact of NBTI and PBTI on SRAM static/dynamic noise margins and cell failure probability," Microelectronics Reliability, Vol 49, pp. 642-649, 2009.

[18] A.E. Islam et. al., "Recent Issues in Negative-Bias Temperature Instability: Initial Degradation, Field Dependence of Interface Trap Generation, Hole Trapping Effects, and Relaxation," Transactions on Electron Devices, Vol. 54, No. 9, pp. 2143-2154, 2007.

[19] S. Mahapatra et. al., "On the Physical Mechanism of NBTI in Silicon Oxynitride p-MOSFETs: Can Differences in Insulator Processing Conditions Resolve the Interface Trap Generation versus Hole Trapping Controversy?" IRPS Tech. Dig., pp. 1-4, 2007.

[20] J.H. Lee et. al., "Separation Method of Hole Trapping and Interface Trap Generation and Their Roles in NBTI Reaction-Diffusion Model," IRPS Tech. Dig., pp. 745-746, 2008.

[21] B. Kaczer et. al., "NBTI from the perspective of defect states with widely distributed time scales," IRPS Tech. Dig., pp. 55-60, 2009.

[22] T. Grasser, et. al., "Switching Oside Traps as the Missing Link between Negative Bias Temperature Instability and Random Telegraph Noise," IEDM Tech. Dig., pp. 729-732, 2009.

[23] E. Hoekstra, "Large Signal Excitation Measurement Techniques for RTS Noise in MOSFETs," EUROCON, pp, 1863-1866, 2005.

[24] M. Denais et. al., "On-the-fly characterization of NBTI in ultra-thin gate oxide PMOSFET's," IEDM Tech. Dig., pp. 109-112, 2004.

[25] H. Aono et. al., "A Study of SRAM NBTI by OTF Measurement," IRPS Tech. Dig., pp. 67-71, 2008. IEDM Tech. Dig., pp. 109-112, 2004.

[26] A.E. Islam, et. al., "On the Differences between Ultra-fast NBTI Measurements and Reaction-Diffution Theory," IEDM Tech. Dig., pp. 733-736, 2009.

[27] T. Nagumo, et. al., "New Analysis Methods for Comprehensive Understanding of Random Telegraph Noise," IEDM Tech. Dig., pp. 759$762,2009$. 